\title{
Editorial: Coarse Graining in Quantum Gravity: Bridging the Gap Between Microscopic Models and Spacetime-Physics
}

\author{
Astrid Eichhorn ${ }^{1 *}$, Benjamin Bahr ${ }^{2}$ and Antonio D. Pereira ${ }^{3,4}$ \\ ${ }^{1}$ CP3-Origins, University of Southern Denmark, Odense, Denmark, ${ }^{2}$ II Institute for Theoretical Physics, University of \\ Hamburg, Hamburg, Germany, ${ }^{3}$ Institute for Mathematics, Astrophysics and Particle Physics, Radboud University, Nijmegen, \\ Netherlands, ${ }^{4}$ Instituto de Física, Universidade Federal Fluminese, Niterói, Brazil
}

Keywords: quantum gravity, renormalization group, asymptotic safety, loop quantum gravity, spin foams, group field theory, causal dynamical triangulations, Horava Lifshitz gravity

\section{Editorial on the Research Topic}

OPEN ACCESS

Edited by:

Jan De Boer,

University of Amsterdam, Netherlands

Reviewed by:

Djordje Minic,

Department of Physics, United States

Daniele Oriti,

Ludwig-Maximilians-University

Munich, Germany

*Correspondence:

Astrid Eichhorn

eichhorn@cp3.sdu.dk

Specialty section:

This article was submitted to

High-Energy and Astroparticle

Physics,

a section of the journal

Frontiers in Physics

Received: 26 March 2021

Accepted: 22 April 2021

Published: 21 May 2021

Citation:

Eichhorn A, Bahr B and Pereira AD (2021) Editorial: Coarse Graining in Quantum Gravity: Bridging the Gap

Between Microscopic Models and

Spacetime-Physics.

Front. Phys. 9:686352.

doi: 10.3389/fphy.2021.686352
Coarse Graining in Quantum Gravity: Bridging the Gap Between Microscopic Models and Spacetime-Physics

Historically, a large number of approaches to quantum gravity take their starting point in the observation that perturbative renormalizability breaks down for the Einstein Hilbert action. This has triggered developments of various paradigms and frameworks that in a more or less radical sense depart from more standard quantum field theoretic ideas. Yet, early on, several ideas already existed on how to formulate quantum gravity as a local quantum field theory with a focus on its Renormalization Group (RG) behavior, including Weinberg's asymptotic-safety idea and Stelle's asymptotically free higher-derivative gravity. Today, the RG is experiencing a renaissance in quantum gravity. This goes significantly beyond the concept of renormalization as a systematic procedure for removing divergences. Rather, the RG plays a central role in the very definition of the quantum theory. In short, technical breakthroughs in various formulations of coarse graining enable a search for universality and scale symmetry in a broad range of setups. In the modern formulation of RG tools for quantum gravity, the notion of scale is implemented in several distinct, intricate ways that are even applicable to a pre-geometric setting.

In summary, a range of distinct approaches to quantum gravity are converging toward the point of view that coarse-graining and the associated notion of scale symmetry could enable us to probe properties of quantum space-time. This convergence in itself could prove to be a catalyst for breakthroughs: while every single approach to quantum gravity is facing open questions and challenges, both of conceptual and of technical nature, many insights obtained within the distinct approaches are in fact complementary. Questions that are seen as technically and/or conceptually challenging in one given approach, might be more easily tackled in another one. Therefore, the development of a common language like the RG and the associated unified conceptual framework holds the promise that important insights could be translated between approaches.

Moreover, there is the distinct possibility that what we now perceive of as different approaches to quantum gravity are in fact simply mathematically different formulations of the same physics. More specifically, different approaches could give rise to the same universality class, thus resulting in the same infrared physics. At the same time, a continuum limit encoded in that universality class could ensure the restoration of diffeomorphism symmetry in discrete approaches. We consider 
the confluence of quantum-gravity approaches at this RGvantage point a promising research area that is still in its relatively early stages. The special issue "Coarse graining in quantum gravity: Bridging the gap between microscopic models and spacetime physics" provides an incomplete snap-shot of this evolving field, highlighting novel ideas, pointing out open challenges and reviewing recent developments. The diverse perspectives brought together in this issue highlight the broad set of research lines converging toward each other as well as the broad range of research opportunities that are opening up.

A series of papers focuses on various aspects of the asymptotic-safety approach, both with continuum and lattice techniques.

In Donoghue provides a constructive criticism of the asymptotic-safety program and discusses a key open question of the current state of the art of the asymptotic safety program, namely the Lorentzian signature of space time. In Bonanno et al. the authors critically reflect on the state of the art in asymptotically safe quantum gravity, providing a comprehensive list of open questions and critically reviewing potential pathways to finding answers both within the functional RG approach and lattice techniques. These two papers exemplify the usefulness of constructive criticism across research lines in quantum gravity.

In Ambjorn et al. use the Causal Dynamical Triangulation approach as a concrete framework to search for asymptotic safety in quantum gravity. They use measurements of the correlation function of the spatial volume profile of the emergent effective spacetime to define lines of constant physics and search for a UV fixed point.

Two papers deal with two aspects of background independence in functional RG techniques for quantum gravity. In Pawlowski and Reichert provide a comprehensive review of fluctuation field calculations in the functional RG framework for asymptotically safe quantum gravity. The fluctuation field arises within the background field methods that enables the definition of a local coarse graining procedure in a background independent manner. In Pagani and Reuter explore the peculiarities and physical implications of backgroundindependent RG flows using field theoretic degrees of freedom like the metric. The requirement of background independence is encoded in self-consistent backgrounds. Both technically and conceptually this constitutes a departure from standard coarse-graining procedures on a fixed background. Specifically, the authors discuss how the so-called naturalness problem regarding the cosmological constant is profoundly different from this perspective.

In Platania reviews developments that tackle a central question in any approach to quantum gravity, namely its phenomenological viability. More specifically, she explores potential consequences of gravitational anti screening, associated with the UV limit in asymptotically safe quantum gravity, in early-universe cosmology. The current state of the art in the field does not yet allow a robust derivation of effects in cosmology from a fundamental gravity theory, instead one can develop and analyze quantum-gravity inspired models. The tentative nature of the link between a fundamental theory and cosmological observations also makes a quantitative comparison between distinct approaches to quantum gravity challenging. In the absence of experimentally measurable observables, the comparison of characteristic properties of the quantum geometry between approaches to quantum gravity is a potential pathway to find commonalities or differences. In this spirit, Kurov and Saueressig link functional RG techniques to the analysis of geometric operators and observables in quantum gravity.

In Held discusses the notion of effective asymptotic safety. This can be viewed as an additional step in Weinberg's translation of interacting RG fixed points from statistical physics, as the asymptotically safe fixed point is approached at scales that are infrared scales when viewed from a more fundamental model (while they remain UV scales compared to, e.g., the electroweak scale). In that scenario, an interacting fixed point is therefore approximately realized over an intermediate regime of scales, focusing trajectories starting from different microphysics onto common predictions for the macrophysics. This qualitative idea-that has the potential to provide a unification between asymptotically safe gravity and other approaches-is made quantitively precise with a calculable notion of predictive power worked out in detail in gauge-Yukawa models.

Other approaches to quantum gravity in which RG ideas and techniques play a role are then discussed in the following list of papers.

In Steinhaus provides an updated review on the implementation of coarse-graining techniques in spin foam models. He focuses on modern techniques such as tensor network renormalization. Such techniques could allow to search for and probe the continuum limit of such models. In the closely related group field theories, in Finocchiaro and Oriti report on the status of simplicial group field theories and their renormalization. In particular, they present a perturbative computation of corrections to correlation functions and provide a road map for the field.

In Thiemann takes the canonical quantum gravity approach as a mathematically well-defined setup to tackle the problem of quantizing gravity. He applies the constructive quantum field theory program and, as such, advocates that renormalization is an essential ingredient to fix some ambiguities in the framework. This constitutes a review of a series of papers written by the author and collaborators, where Hamiltonian Renormalization is established in the canonical language by means of constructive quantum field theory. One of the papers in that series is also published in the present special issue, see by Liegener and Thiemann.

A different solution to the coarse-graining problem in gravity is implemented in the discrete, pre-geometric setting of matrix models by Castro and Koslowski. In Castro and Koslowski, they apply discrete functional RG techniques to matrix models which encode a preferred foliation. They discover fixed points which are compatible with known results from causal dynamical triangulations in two dimensions and thereby establish the applicability of these techniques in a causal setting. This work constitutes a compelling example of how numerical Monte-Carlo simulations can meet the computationally less expensive techniques arising from the functional RG. 
In Steinwachs reviews developments that aim at formulating quantum gravity as a perturbatively renormalizable and ghost-free quantum field theory, enabled by a breaking of full diffeomorphism invariance to foliation-preserving diffeomorphisms.

In summary, these papers provide a partial snapshot of the state-of-the-art of the RG framework to quantum gravity that brings together previously disconnected approaches and is suitable to tackle both formal as well as phenomenological questions.

\section{AUTHOR CONTRIBUTIONS}

All authors listed have made a substantial, direct and intellectual contribution to the work, and approved it for publication.

\section{FUNDING}

AP acknowledges CNPq under the grant PQ-2 (309781/2019-1), FAPERJ under the "Jovem Cientista do Nosso Estado" program (E26/202.800/2019), and NWO under the VENI Grant (VI.Veni.192.109) for financial support.

Conflict of Interest: The authors declare that the research was conducted in the absence of any commercial or financial relationships that could be construed as a potential conflict of interest.

Copyright (c) 2021 Eichhorn, Bahr and Pereira. This is an open-access article distributed under the terms of the Creative Commons Attribution License (CC BY). The use, distribution or reproduction in other forums is permitted, provided the original author(s) and the copyright owner(s) are credited and that the original publication in this journal is cited, in accordance with accepted academic practice. No use, distribution or reproduction is permitted which does not comply with these terms. 